\title{
OPTICALLY DETECTED CYCLOTRON RESONANCE STUDIES OF CdMnTe/CdTe MULTIQUANTUM WELLS AND CdMgTe/CdMnTe SUPERLATTICES
}

\author{
M. GodLEWSKI, K. ŚWIĄTEK \\ Institute of Physics, Polish Academy of Sciences \\ Al. Lotników 32/46, 02-668 Warszawa, Poland \\ C.I. Harris, J.P. Bergman, B. Monemar \\ Department of Physics and Measurement Technology, Linköping University \\ 58183 Linköping, Sweden \\ AND A. WAAG. \\ Institute of Physics, Würzburg University, Am Hubland, 8700 Würzburg, Germany \\ The results of photoluminescence, time resolved photoluminescence (de- \\ cay times), optically detected cyclotron and magnetic resonances investiga- \\ tions of CdMnTe/CdTe multiquantum wells and CdMgTe/CdMnTe super- \\ lattices are presented. The role of defects and quantum well width fluctua- \\ tions in recombination processes of $2 \mathrm{D}$ carriers is discussed.
}

PACS numbers: 68.55.Ln, 71.35.+z, 76.70.Hb, 78.47.+p

\section{Introduction}

It was recently realized that defects can influence low temperature recombination processes in nominally undoped wide quantum wells (QWs) of GaInAs [1]. In this communication we discuss the role of defects and potential fluctuations (due to QW width fluctuations) on carrier localization and scattering processes in molecular beam epitaxy (MBE) grown MQWs (multiquantum wells) of $\mathrm{CdTe} / \mathrm{CdMnTe}$ and superlattices (SLs) of CdMgTe/CdMnTe. The dynamics of exciton recombination in QWs is also discussed and a new application of the optically detected cyclotron resonance (ODCR) method is presented. 


\section{Results and discussion}

A time integrated photoluminescence (PL) spectrum of $\mathrm{CdTe} / \mathrm{CdMnTe}$ MQW structure is shown in Fig. 1a. The sample studied was grown by MBE on a (100) CdTe substrate with a $1 \mu \mathrm{m}$ CdTe buffer layer, followed by a $6000 \AA \mathrm{CdMnTe}$ layer, and then six CdTe QWs of 6,12,18, 28,60 and $100 \AA$ width separated by $500 \AA \mathrm{CdMnTe}$ barriers covered with $1000 \AA \mathrm{CdMnTe}$ cap layer. The Mn fraction in the CdMnTe barrier was about $10 \%$.

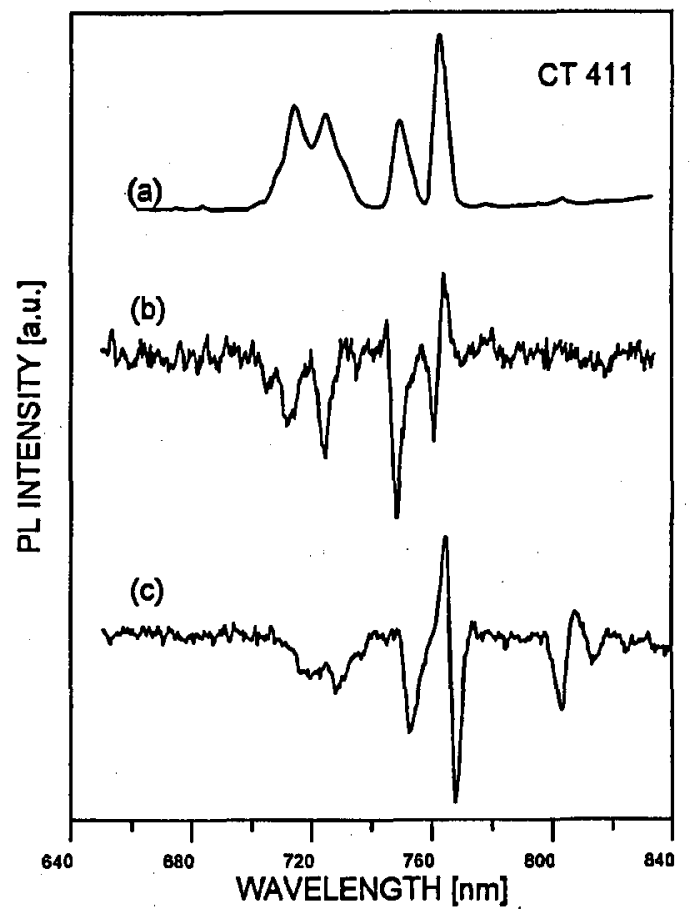

Fig. 1. Low resolution PL spectrum of CdTe/CdMnTe MQW system (a). In (b), (c) the spectral dependencies of the ODCR signal (ODCR-PL) are shown. The ODCR-PL spectra were measured in phase with on-off modulated microwaves with magnetic field set at cyclotron resonance. The (c) spectrum was recorded for lower excitation intensity than used in the case of (b).

PL kinetics were measured for $100 \AA \mathrm{QW}$ and $60 \AA \mathrm{QW}$ emissions. The measured PL kinetics shows that for both 60 and $100 \AA$ QWs the emission consists of two recombination bands (not resolved in the stationary PL experiment) of different rise and decay time. The higher energy band rises within $50 \mathrm{ps}$ time and does not shift its spectral position during the decay time, i.e., there is no evidence of exciton diffusion to sites of lower energies (larger QW width). The second band (down-shifted by about 2-3 meV) rises in $80 \mathrm{ps}$ time and has a longer decay time. This band, attributed to the donor bound exciton (DBE) emission, shifts towards 
lower energies by about $0.6 \mathrm{meV}$ during the time of the decay. The present results confirm the kinetics measurements recently performed on similar structures [2], but differ considerably from those observed for III-V QW structures, where a larger shift of the exciton bands is observed during the decay [3]. The PL decay experiments indicate that the high energy PL band has properties of a localized exciton (LE) emission, and that the LE is trapped by potential fluctuations in the QW. The shift between PL excitation maximum and the LE maximum is about $2 \mathrm{meV}$, which indicates high quality of the structure studied. The lower energy band, attributed to the DBE emission, can be saturated at increased laser power. The PL kinetics measurements indicate that there is an energy transfer link between the LE and $\mathrm{BE}$ emissions.

The ODCR technique can be used to estimate the magnitude of the potential fluctuations in a QW [4]. The response of the inhomogeneously broadened LE and DBE emissions to carrier heating under cyclotron resonance (CR) conditions was studied. Electron and heavy hole CRs were observed in the ODCR, via the decrease in the intensity of the LE emission from the CdTe quantum well. A very strong quantum confinement effect was observed. Relatively well resolved CRs were observed only for a magnetic field normal to the heterointerfaces. For a tilted magnetic field an approximately linear decrease in QW emission intensity vs. magnetic field magnitude was observed. The latter effect is known from previous studies and may result in the so-called magnetic freeze-out of carriers from QW [5] and a type $\mathrm{I} \rightarrow$ type II transition for the valence band confinement potential [6]. The mobilities derived from the width of the electron $\left(4 \times 10^{5} \mathrm{~cm}^{2} /(\mathrm{V} \mathrm{s})\right)$ and heavy hole $\left(5 \times 10^{4} \mathrm{~cm}^{2} /(\mathrm{V} \mathrm{s})\right)$ CRs are very high and are similar to those observed in ODCR studies of high quality bulk CdTe [7]. The high mobility of the carriers derived from ODCR study of bulk CdTe was explained by photoneutralization and/or screening of ionized impurities [7]. The present ODCR study indicates that even for high quality nominally undoped wide QWs scattering at ionized impurities may control 2D carrier mobilities.

The observation of $\mathrm{CR}$ is related to carrier heating at resonance leading to characteristic hot carrier effects on exciton emissions [7]. Heating of carriers participating in exciton formation prevents their binding and results in a decrease in intensity of the exciton emission. The spectral dependence of CR signals is shown in Fig. 1b, c. The ODCR results indicate that at increased microwave power hot carriers can diffuse out from the QWs to the substrate. This effect dominates for the narrower QWs. For the two wider QWs carrier heating under CR conditions results in a derivative-like response of the emission bands. Two different such PL responses are depicted in Fig. $1 \mathrm{~b}$ and c. A positive component was observed on the high energy part of ODCR-PL spectrum of the $60 \AA \mathrm{QW}$, this indicates that LEs can be turned into free excitons (FEs) by interaction with hot carriers. A small shift between LE and FE PL bands confirms previous estimations that the fluctuations of the QW width are restricted to 1 monolayer [2]. For the $100 \AA \mathrm{QW}$ the higher energy part of the PL spectrum is quenched, whereas the lower is enhanced due to the differenee in carrier localization energy (exciton binding energy) for QW regions of different average width. Hot carriers impact ionizes excitons in the regions of lower binding energy, which in turn enhances the recombination rates 
in regions of higher binding energy (larger average $\mathrm{QW}$ width). The small shift observed for the $100 \AA \mathrm{QW}$ also confirms the small magnitude (between 0.5 and 1 monolayer) of the width fluctuations in the QW system studied.

For lower excitation intensity the DBE emission contributes to PL from the wider QWs. The observed response of the $100 \AA$ QW PL (Fig. 1c) can be explained by impact ionization of the DBEs, which enhances the LE/FE recombination. For "magnetic" QWs in the CdMgTe/CdMnTe system the ODCR experiment indicates that the magnitude of the potential fluctuations in QWs is enhanced due to the fluctuations of the magnetization caused by inhomogeneities in the Mn fraction in the QW.

\section{References}

[1] P. Omling, Appl. Phys. Lett. 59, 2022 (1991).

[2] A. Waag, S. Schmeusser, R.N. Bicknell-Tassius, D.R. Yakovlev, W. Ossau, G. Landwehr, I.N. Uraltsev, Appl. Phys. Lett. 59, 2995 (1991).

[3] M. Zachau, J.A. Kash, W.T. Masselink, Phys. Rev. B 44, 8403 (1991).

[1] B.M. Ashkinadze, E. Cohen, A. Ron, L. Pfeiffer, Phys. Rev. B 47, 10613 (1993).

[5] S. Datta, J.K. Furdyna, R.L. Gunshor, Superlattices Microstruct. 1, 327 (1985).

[6] X. Liu, A. Petrou, J. Warnock, B.T. Jonker, G.A. Prinz, J.J. Krebs, Phys. Rev. Lett. 3, 2280 (1989).

[7] R. Romestain, C. Weisbuch, Phys. Rev. Lett. 45, 2067 (1980). 